J. Nonlinear Var. Anal. 5 (2021), No. 6, pp. 929-938

Available online at http://jnva.biemdas.com

https://doi.org/10.23952/jnva.5.2021.6.06

\title{
CONVERGENCE ANALYSIS OF A PROJECTED GRADIENT METHOD FOR MULTIOBJECTIVE OPTIMIZATION PROBLEMS
}

\author{
XIAOPENG ZHAO ${ }^{1}$, QIAOYUE SUN ${ }^{1}$, LIYA LIU $^{2}$, SUN YOUNG CHO ${ }^{3, *}$ \\ ${ }^{1}$ School of Mathematical Sciences, Tiangong University, Tianjin 300387, China \\ ${ }^{2}$ School of Mathematics and Statistics, Southwest University, Chongqing 400715, China \\ ${ }^{3}$ Research Center for Interneural Computing, China Medical University Hospital, \\ China Medical University, Taichung 40402, Taiwan
}

\begin{abstract}
In this paper, we consider a projected gradient method for constrained multiobjective optimization problems. Under suitable assumptions, we show that the sequence generated by the method converges to a Pareto stationary point (or a weak Pareto optimal point) of the problem when the multiobjective function is quasiconvex (or pseudoconvex). Furthermore, in the case that the multiobjective function is convex, by using some approximate conditions that are imposed on the gradients of the objective functions and the search directions, we obtain the linear convergence result for this method.
\end{abstract}

Keywords. Linear convergence; Multiobjective optimization; Projected gradient method; Pareto optimality.

\section{INTRODUCTION}

In multiobjective optimization, one needs to minimize or maximize several objective functions at the same time. This formulism recasts numerous problems in various areas, including engineering, economy, design, statistics, management science and so on; see, e.g., [1, 2, 3, 4, $5,6,7]$. Since the points that optimize all the given objective functions simultaneously do not exist necessarily, one usually uses the concept of the Pareto optimality.

In this paper, we center on a numerical algorithm, i.e., the projected gradient method, for solving multiobjective optimization problems. As a classical and efficient method, the projected gradient method and its variant were extensively investigated by researchers for solving scalar optimization problems $[8,9,10,11,12,13,14]$, and they were further extended to the multiobjective optimization setting successfully; see, e.g., $[15,16,17,18]$ and the references therein.

In [17], Graña Drummond and Iusem considered a projected gradient method for constrained vector optimization problems, and showed that every accumulation point (if any) of the sequence generated by this method is a stationary point. Afterwards, Fukuda and Graña Drummond established in [19] the full convergence of the sequence to optimal points for convex

\footnotetext{
${ }^{*}$ Corresponding author.

E-mail addresses: zhaoxiaopeng.2007@163.com (X. Zhao), tjgd140024@163.com (Q. Sun), liya42@ @ swu.edu. cn (L. Liu), ooly61@ hotmail.com (S.Y. Cho).

Received May 1, 2021; Accepted July 24, 2021.
}

(C)2021 Journal of Nonlinear and Variational Analysis 
objective functions. Note that, in many real-life applications, such as, microeconomy and location theory $[20,21]$, one often encounters the situations where the objective function is not convex, e.g. is quasiconvex. Therefore, the study of nonconvex multiobjective optimization problems is of great theoretical significance and practical application value. For this, in [15], Bello Cruz et al. studied the projected gradient method of [17] for solving constrained quasiconvex multiobjective optimization problem. By exploiting the structure of quasiconvexity of the problem, they obtained the convergence of the sequence generated by the algorithm to a stationary point, and further to a weak Pareto optimal point when the multiobjective function is assumed to be pseudoconvex.

Recently, Fazzio and Schuverdt [16] extended this projected gradient method to include a nonmonotone line search for constrained convex multiobjective optimization problems. They associated a variable steplength instead of the original fixed parameter to compute the search direction. For this method, they showed the stationarity of the cluster points (without convexity assumption) and proved the convergence of the sequence generated by the algorithm to a weak Pareto optimal point (under the convexity assumption).

Inspired by these works, in this paper, we develop the method presented in [15] to include variable parameter instead of the fixed one for the constrained quasiconvex multiobjective optimization problem. Under suitable assumptions, we show that the sequence produced by our algorithm converges to a stationary point (or a weak Pareto optimal point) in the case that the multiobjective function is quasiconvex (or pseudoconvex).

In addition, notice that, in most of the existing works, only the strong convergence property of iterative sequences were considered. While, from the perspective of high efficiency in solving practical problems, it is very necessary to obtain better convergence properties, for example the linear convergence property. However, at present, there is not much work on this topic in multiobjective optimization. In [22], Mahdavi-Amiri and Salehi Sadaghiani proposed a quasi-Newton method for unconstrained strongly convex multiobjective optimization problems and obtained the local superlinear rate of convergence for the method. Qu et al. [23] considered projected gradient methods for convex vector optimization and analyzed the local linear convergence rate for the proposed methods.

Motivated by these results, in this paper, we aim to establish the full linear convergence result of the method considered here. Observe that, in the scalar optimization, Zhang and Hager [9] proposed a nonmonotone gradient algorithm for the unconstrained optimization problems. Under some appropriate assumptions of the gradients of objective functions and the search directions, they established the linear convergence for their algorithm in the case that the objective function is strongly convex. In this paper, we extend the techniques that used in [9] to the case of the multiobjective optimization. Specifically, by making some changes to the assumptions, we obtain the linear convergence result of the full sequence generated by our method when the multiobjective function is only convex, which is one of the highlights of this paper.

The paper is organized as follows. In Section 2, we recall some notations and preliminary results. In Section 3, we give the algorithm and present some related properties that will be used later. Section 4, the last section, contains the main results, where the convergence analysis of the algorithm is provided in the case that the mutiobjective function is quasiconvex and convex, respectively. 


\section{PRELIMINARIES}

In this section, we recall some necessary notations and results (see, e.g., $[4,24,25])$ that are used throughout the paper. By $\langle\cdot, \cdot\rangle$, we denote the inner product on $\mathbb{R}^{n}$, and by $\|\cdot\|$ we denote the corresponding norm. The transpose sign is denoted by $\top$. As usual, $\mathbb{N}$ denotes the set of all positive integers, and let $\mathbb{N}^{*}=\mathbb{N} \cup\{0\}$. Let $\mathbb{R}_{+}^{m}$, respectively, $\mathbb{R}_{++}^{m}$ denote the nonnegative orthant, respectively, the positive orthant of $\mathbb{R}^{m}$. We consider the partial orders $\preceq(\prec)$, defined as $u \preceq v(u \prec v)$ if and only if $v-u \in \mathbb{R}_{+}^{m}\left(v-u \in \mathbb{R}_{++}^{m}\right)$.

Let $C \subseteq \mathbb{R}^{n}$ be a closed and convex set, and let $F$ be a vector-valued function given by $F:=\left(f_{1}, \cdots, f_{m}\right)^{\top}$ with each $f_{i}$ being a continuously differentiable real-valued function on an open superset of $C$. In this paper, we consider the following constrained multiobjective optimization problem:

$$
\text { Minimize } F(x), \quad \text { subject to } x \in C \text {. }
$$

We use $J_{F}\left(x^{*}\right):=\left(\nabla f_{1}\left(x^{*}\right), \cdots, \nabla f_{m}\left(x^{*}\right)\right)^{\top}$ to denote the Jacobian matrix of $F$ at $x^{*} \in \mathbb{R}^{n}$, where each $\nabla f_{i}\left(x^{*}\right)$ with $i=1, \cdots, m$ is the gradient of $f_{i}$ at $x^{*}$.

Definition 2.1. A point $x^{*} \in C$ is called

(a) a Pareto stationary point (or Pareto critical point) of problem (2.1) if

$$
J_{F}\left(x^{*}\right)\left(C-x^{*}\right) \cap\left(-\mathbb{R}_{++}^{m}\right)=\emptyset,
$$

(b) a weak Pareto optimal point (or weak Pareto efficient solution) of problem (2.1) if there does not exist $x \in C$ such that $F(x) \prec F\left(x^{*}\right)$,

(c) a Pareto optimal point (or Pareto efficient solution) of problem (2.1) if there does not exist $x \in C$ such that $F(x) \preceq F\left(x^{*}\right)$ and $F(x) \neq F\left(x^{*}\right)$.

It is well-known that every Pareto optimal point is also a weak Pareto optimal point, and every weak Pareto optimal point is also a Pareto stationary point. But, in general, the converse may not be true.

Recall that a real-valued function $f: \mathbb{R}^{n} \rightarrow \mathbb{R}$ is said to be convex, respectively, quasiconvex if, for any $x, y \in \mathbb{R}^{n}$ and $t \in[0,1]$,

$$
f(t x+(1-t) y) \leq t f(x)+(1-t) f(y)
$$

respectively,

$$
f(t x+(1-t) y) \leq \max \{f(x), f(y)\} .
$$

When $f$ is differentiable, $f$ is quasiconvex if and only if, for any $x, y \in \mathbb{R}^{n}, f(x) \leq f(y)$ implies that $\langle\nabla f(y), x-y\rangle \leq 0$; see [26].

A differentiable function $f: \mathbb{R}^{n} \rightarrow \mathbb{R}$ is said to be pseudoconvex if and only if $\langle\nabla f(y), x-$ $y\rangle \geq 0$ implies that $f(x) \geq f(y)$. It is known that convex (or pseudoconvex) functions are quasiconvex.

We say that the multiobjective function $F$ is convex (quasiconvex, or pseudoconvex) if each component function $f_{i}, i=1, \cdots, m$, of $F$ is convex (quasiconvex, or pseudoconvex). In the case when $F$ is convex (or pseudoconvex), it can be verified by definition (one also can refer to $[15,17])$ that a Pareto stationary point of $(2.1)$ is also a weak Pareto optimal point. However, usually this result does not hold for quasiconvex functions (see [27]). 
A scalar function $f: \mathbb{R}^{n} \rightarrow \mathbb{R}$ is said to be Lipschitz continuous with Lipschitz constant $L \in[0,+\infty)$ if

$$
|f(x)-f(y)| \leq L\|x-y\|, \quad \text { for all } x, y \in \mathbb{R}^{n} .
$$

In this paper, we will discuss the linear convergence: a sequence $\left\{u_{k}\right\} \subseteq \mathbb{R}^{n}$ is said to converge linearly to its limit $u$ with rate $r$ if $r \in[0,1)$ and there is some $a \geq 0$ such that

$$
\left\|u_{k}-u\right\| \leq a r^{k} \quad \text { for all } k \in \mathbb{N} .
$$

Finally, we present the following quasi-Fejér convergence theorem, which has been applied extensively by many authors to analyze the convergence of the gradient methods; see, e.g., $[15,16,17,18,19]$ and the references therein. Recall that a sequence $\left\{u_{k}\right\} \subseteq \mathbb{R}^{n}$ is said to be quasi-Fejér convergent to a nonempty set $U \subseteq \mathbb{R}^{n}$ if, for each $u \in U$, there exists a sequence $\left\{\varepsilon_{k}\right\} \subseteq \mathbb{R}_{+}$such that $\sum_{k=0}^{\infty} \varepsilon_{k}<\infty$ and

$$
\left\|u_{k+1}-u\right\|^{2} \leq\left\|u_{k}-u\right\|^{2}+\varepsilon_{k} \quad \text { for all } k \in \mathbb{N} .
$$

Proposition 2.1. ([28, Theorem 1]) If $\left\{u_{k}\right\} \subseteq \mathbb{R}^{n}$ is quasi-Fejér convergent to a nonempty set $U \subseteq \mathbb{R}^{n}$, then $\left\{u_{k}\right\}$ is bounded. Furthermore, if an accumulation point $\bar{u}$ of $\left\{u_{k}\right\}$ belongs to $U$, then $\left\{u_{k}\right\}$ is convergent to $\bar{u}$.

\section{The Algorithm}

We describe in this section the algorithm here for constrained multiobjective optimization problem (2.1).

Algorithm 3.1. Initialization: Choose the parameters $\sigma \in(0,1), v>1,0<\beta_{\min }<\beta_{\max }<\infty$ and $\beta_{0} \in\left[\beta_{\min }, \beta_{\max }\right]$. Let $x_{0} \in C$ be an arbitrary initial iterative point. Set $k=0$.

Step 1 Compute the search direction

$$
v_{k}:=\operatorname{argmin}_{v \in C-x_{k}} \varphi_{k}(v),
$$

where $\varphi_{k}(v)=\beta_{k} \max _{1 \leq i \leq m}\left\langle\nabla f_{i}\left(x_{k}\right), v\right\rangle+\frac{\|v\|^{2}}{2}$.

If $v_{k}=0$, then stop. Otherwise,

Step 2 Compute

$$
j(k):=\min \left\{j \in \mathbb{N}^{*}: F\left(x_{k}+v^{-j} v_{k}\right) \preceq F\left(x_{k}\right)+\sigma v^{-j} J_{F}\left(x_{k}\right) v_{k}\right\} .
$$

Step 3 Define

$$
x_{k+1}=x_{k}+\gamma_{k} v_{k},
$$

where $\gamma_{k}=2^{-j(k)}$. Set $k=k+1$ and go back to Step 1 .

In the case that the variable parameter $\beta_{k}$ is a constant and $v=2$, Algorithm 3.1 reduces to the one proposed by Bello Cruz et al. [15]. In [15], they considered the strong convergence results for the algorithm therein. While, in this paper, in addition to the strong convergence property, we will further investigate the linear convergence of Algorithm 3.1.

Note that, for each $k$, the direction $v_{k}$ can be obtained due to the strong convexity of the function $\varphi_{k}$. If $v_{k} \neq 0$, then $\varphi_{k}\left(v_{k}\right)<0$, which implies that $J_{F}\left(x_{k}\right) v_{k} \prec 0$. Whence, [17, Proposition 1] ensures that the Armijo-like line search rule (3.1) in Step 2 is well-defined and so is the whole algorithm. From (3.1), we can also obtain that the sequence of objective function values $\left\{F\left(x_{k}\right)\right\}$ is monotone decreasing in the partial order, i.e., $F\left(x_{k+1}\right) \preceq F\left(x_{k}\right)$ for all $k$. Besides, the 
feasibility of the sequence $\left\{x_{k}\right\}$ generated by Algorithm 3.1 (i.e., $\left\{x_{k}\right\} \subseteq C$ ) can be verified by the definition of the algorithm. We summarize these conclusions as the following proposition.

Proposition 3.1. The sequence $\left\{x_{k}\right\}$ generated by Algorithm 3.1 is feasible, and the sequence $\left\{F\left(x_{k}\right)\right\}$ is monotone decreasing.

By the classical characterization of stationary points in [17, Proposition 3], we have that $x_{k}$ is a Pareto stationary point for problem (2.1) if Algorithm 3.1 stops at some iteration $k$ with the stopping criteria in Step 1. From now on, we assume that the sequence $\left\{x_{k}\right\}$ generated by Algorithm 3.1 is infinite. In the end of this section, we give the following sequence inequality. Since it can be shown by using the similar argument as that in [15, Lemma 2], we omit the proof here.

Lemma 3.1. For all $x \in C$ and $k \in \mathbb{N}$, it holds that

$$
\left\|x_{k+1}-x\right\|^{2} \leq\left\|x_{k}-x\right\|^{2}+2 \gamma_{k} \beta_{k}\left\langle\sum_{j=1}^{m} \lambda_{j}^{k} \nabla f_{j}\left(x_{k}\right), x-x_{k}\right\rangle+\frac{2 \beta_{k}}{\sigma} \sum_{j=1}^{m}\left(f_{j}\left(x_{k}\right)-f_{j}\left(x_{k+1}\right)\right),
$$

where $\left\{\lambda_{j}^{k}\right\}_{j=1}^{m} \subseteq[0,1]$ with $\sum_{j=1}^{m} \lambda_{j}^{k}=1$.

\section{Convergence Analysis}

In this section, we discuss the convergence of the sequence $\left\{x_{k}\right\}$ generated by Algorithm 3.1 in different situations. Specifically, in the case that the multiobjective function is quasiconvex, we show $\left\{x_{k}\right\}$ converges to a stationary point of (2.1). As a natural consequence, the convergence of $\left\{x_{k}\right\}$ to a weak Pareto optimal point of (2.1) can be obtained when the multiobjective function is pseudoconvex. Then, we consider the case that the multiobjective function is convex and prove the global linear convergence result for the algorithm.

In this section, we need the following assumption for our convergence analysis.

(A1) The set $T:=\left\{x \in C: F(x) \preceq F\left(x_{k}\right), \forall k \in \mathbb{N}\right\}$ is nonempty.

This assumption has been widely used to analyze the convergence of many numerical methods for vector optimization problems, for example the projected gradient method $[15,17,18$, 19], steepest descent method [28, 29] and the proximal point method [21, 30].

4.1. The quasiconvex case. Let us start with the following result, which only requires the continuous differentiability of $F$.

Lemma 4.1. Assume that (A1) holds. Then, every accumulation point, if any, of the sequence $\left\{x_{k}\right\}$ is a Pareto stationary point of problem (2.1).

Proof. This result can be obtained from [16, Theorem 1] when the nomonotone line search rule considered therein reduces to the Armijo-like line search rule (3.1).

Next, we study the convergence property of Algorithm 3.1 when the multiobjective function $F$ is quasiconvex. The following result is the main result in this subsection, which provides a sufficient condition for $\left\{x_{k}\right\}$ to converge to a Pareto stationary point of (2.1). The proof for Theorem 4.1 is similar to that for [15, Theorem 1], but for the sake of completeness, we still give its proof here. 
Theorem 4.1. Assume that (A1) holds and that the function $F$ is quasiconvex. Then, $\left\{x_{k}\right\}$ converges to a Pareto stationary point of problem (2.1).

Proof. Take $x \in T$. For each $i=1, \cdots, m$ and $k$, one has $f_{i}(x) \leq f_{i}\left(x_{k}\right)$. Thus, by the classical characterization of the differentiable quasiconvex function (see [26]), we have that $\left\langle\nabla f_{i}\left(x_{k}\right), x-\right.$ $\left.x_{k}\right\rangle \leq 0$ for all $i=1, \cdots, m$ and $k$. Combining this with Lemma 3.1, one can obtain that

$$
\left\|x_{k+1}-x\right\|^{2} \leq\left\|x_{k}-x\right\|^{2}+\frac{2 \beta_{k}}{\sigma} \sum_{j=1}^{m}\left(f_{j}\left(x_{k}\right)-f_{j}\left(x_{k+1}\right)\right) \quad \text { for all } k \text {. }
$$

Denote

$$
\varepsilon_{k}:=\frac{2 \beta_{k}}{\sigma} \sum_{j=1}^{m}\left(f_{j}\left(x_{k}\right)-f_{j}\left(x_{k+1}\right)\right), \quad \forall k \in \mathbb{N} .
$$

Then, $\left\{\varepsilon_{k}\right\} \subseteq \mathbb{R}_{+}$, which is due to the monotone decreasing property of $\left\{F\left(x_{k}\right)\right\}$ that claimed in Proposition 3.1.

Next, we show that $\sum_{k=0}^{\infty} \varepsilon_{k}<\infty$. Indeed, for any fixed $N \in \mathbb{N}$, we have

$$
\begin{aligned}
\sum_{k=0}^{N} \varepsilon_{k} & =\sum_{k=0}^{N}\left(\frac{2 \beta_{k}}{\sigma} \sum_{j=1}^{m}\left(f_{j}\left(x_{k}\right)-f_{j}\left(x_{k+1}\right)\right)\right) \\
& \leq \frac{2 \beta_{\max }}{\sigma} \sum_{j=1}^{m}\left(\sum_{k=0}^{N}\left(f_{j}\left(x_{k}\right)-f_{j}\left(x_{k+1}\right)\right)\right) \\
& =\frac{2 \beta_{\max }}{\sigma} \sum_{j=1}^{m}\left(f_{j}\left(x_{0}\right)-f_{j}\left(x_{N+1}\right)\right) \\
& \leq \frac{2 \beta_{\max }}{\sigma} \sum_{j=1}^{m}\left(f_{j}\left(x_{0}\right)-f_{j}(x)\right)
\end{aligned}
$$

where the first inequality is obtained from the boundedness of the parameters $\left\{\beta_{k}\right\}$, and the last inequality is derived from assumption (A1).

Therefore, $\left\{x_{k}\right\}$ is quasi-Fejér convergent to $T$. With the aid of Proposition 2.1, we conclude that $\left\{x_{k}\right\}$ is bounded. Let $x^{*}$ be an accumulation point of $\left\{x_{k}\right\}$. Then, it follows from Lemma 4.1 that $x^{*}$ is a Pareto stationary point of (2.1). Moreover, the monotone decreasing property of $\left\{F\left(x_{k}\right)\right\}$ implies that $x^{*} \in T$. By applying Proposition 2.1 again, the convergence of $\left\{x_{k}\right\}$ to $x^{*}$ is followed. The proof is complete.

As we mentioned in Section 2 that the pseudoconvex functions are also quasiconvex, and that when $F$ is pseudoconvex, the Pareto stationary point of (2.1) is also the weak Pareto optimal point. Thus, the following result can be deduced immediately from Theorem 4.1.

Corollary 4.1. Assume that (A1) holds and that the function $F$ is pseudoconvex. Then, $\left\{x_{k}\right\}$ converges to a weak Pareto optimal point of problem (2.1).

4.2. The convex case. In this subsection, we consider the multiobjective optimization problem (2.1) with $F$ being convex. We aim to establish the linear convergence result of Algorithm 3.1. That is, under some approximate assumptions on the gradients of the objective functions and the search directions, the sequence $\left\{F\left(x_{k}\right)\right\}$ converges linearly to the optimal value of problem (2.1).

We begin with the following lemma, in which a lower bound for the step length $\left\{\gamma_{k}\right\}$ is given. 
Lemma 4.2. For any $k$, suppose that each $\nabla f_{i}, i=1, \cdots, m$, satisfies the following Lipschitz condition with Lipschitz constant $L$ in the case that $\gamma_{k}<\frac{1}{v}$ :

$$
\left\|\nabla f_{i}(x)-\nabla f_{i}\left(x_{k}\right)\right\| \leq L\left\|x-x_{k}\right\|
$$

for all $x$ on the line segment connecting $x_{k}$ and $x_{k}+v \gamma_{k} v_{k}$. Then

$$
\gamma_{k} \geq \min \left\{\frac{1}{v}, \frac{1-\sigma}{L v \beta_{\max }}\right\} \quad \text { for all } k \in \mathbb{N} .
$$

Proof. Fix $k \in \mathbb{N}$. Clearly, (4.1) is natural when $\gamma_{k} \geq \frac{1}{v}$. So, in the remainder part of the proof, we consider the case that $\gamma_{k}<\frac{1}{v}$. Since $j(k)$ is the smallest integer such that $\gamma_{k}=v^{-j(k)}$ satisfies the Armijo-like line search in (3.1), we have

$$
F\left(x_{k}+v \gamma_{k} v_{k}\right)=F\left(x_{k}+v^{-(j(k)-1)} v_{k}\right) \npreceq F\left(x_{k}\right)+\sigma v \gamma_{k} J_{F}\left(x_{k}\right) v_{k} .
$$

So, there exists $i_{k} \in\{1, \cdots, m\}$ such that

$$
f_{i_{k}}\left(x_{k}+v \gamma_{k} v_{k}\right)>f_{i_{k}}\left(x_{k}\right)+\sigma v \gamma_{k}\left\langle\nabla f_{i_{k}}\left(x_{k}\right), v_{k}\right\rangle .
$$

Using the Lipschitz continuity assumption on $\nabla f_{i_{k}}$, we obtain

$$
\begin{aligned}
f_{i_{k}}\left(x_{k}+v \gamma_{k} v_{k}\right)-f_{i_{k}}\left(x_{k}\right) & =v \gamma_{k}\left\langle\nabla f_{i_{k}}\left(x_{k}\right), v_{k}\right\rangle+\int_{0}^{v \gamma_{k}}\left\langle\nabla f_{i_{k}}\left(x_{k}+t v_{k}\right)-\nabla f_{i_{k}}\left(x_{k}\right), v_{k}\right\rangle d t \\
& \leq v \gamma_{k}\left\langle\nabla f_{i_{k}}\left(x_{k}\right), v_{k}\right\rangle+\int_{0}^{v \gamma_{k}} L\left\|v_{k}\right\|^{2} t d t \\
& =v \gamma_{k}\left\langle\nabla f_{i_{k}}\left(x_{k}\right), v_{k}\right\rangle+\frac{L v^{2} \gamma_{k}^{2}\left\|v_{k}\right\|^{2}}{2} .
\end{aligned}
$$

This, together with (4.2), implies

$$
\gamma_{k} \geq \frac{2(\sigma-1)\left\langle\nabla f_{i_{k}}\left(x_{k}\right), v_{k}\right\rangle}{L v\left\|v_{k}\right\|^{2}} .
$$

Moreover, note that $0 \in C-x_{k}$, which is due to the feasibility of $\left\{x_{k}\right\}$. So,

$$
\varphi_{k}\left(v_{k}\right)=\beta_{k} \max _{1 \leq i \leq m}\left\langle\nabla f_{i}\left(x_{k}\right), v_{k}\right\rangle+\frac{\left\|v_{k}\right\|^{2}}{2} \leq 0=\varphi_{k}(0) .
$$

From this and the fact that $\beta_{k} \leq \beta_{\max }$, we have

$$
\left\langle\nabla f_{i}\left(x_{k}\right), v_{k}\right\rangle \leq-\frac{\left\|v_{k}\right\|^{2}}{2 \beta_{k}} \leq-\frac{\left\|v_{k}\right\|^{2}}{2 \beta_{\max }} \quad \text { for each } i=1, \cdots, m .
$$

Combining this with (4.3) gives $\gamma_{k} \geq \frac{1-\sigma}{L v \beta_{\max }}$. This obtains conclusion (4.1) immediately.

To show our convergence result, the following assumption on the search directions is needed.

(A2) There exists a positive constant $c$ such that

$$
\left\langle\nabla f_{i}\left(x_{k}\right), v_{k}\right\rangle \leq-c\left\|\nabla f_{i}\left(x_{k}\right)\right\|, \quad \text { for each } i=1, \cdots, m \text { and } k \in \mathbb{N} .
$$

The main result in this subsection is presented as follows. 
Theorem 4.2. Assume that (A1) and (A2) hold, and each $\nabla f_{i}$ with $i=1, \cdots, m$ is Lipschitz continuous on bounded sets. Then, there exits $\theta \in(0,1)$ such that

$$
F\left(x_{k}\right)-F\left(x^{*}\right) \preceq \theta^{k}\left(F\left(x_{0}\right)-F\left(x^{*}\right)\right) \quad \text { for each } k \in \mathbb{N},
$$

where $x^{*}:=\lim _{k \rightarrow \infty} x_{k}$ is a weak Pareto optimal point of problem (2.1).

Proof. Notice that the convex function is quasiconvex, and the Pareto stationary point of (2.1) is also a weak Pareto optimal point when $F$ is convex. Then, it follows from Theorem 4.1 that $\left\{x_{k}\right\}$ is convergent (so is bounded) and $x^{*}=\lim _{k \rightarrow \infty} x_{k}$ is a weak Pareto optimal point of problem (2.1). Moreover, from (4.4), it can be obtained that, for each $i=1, \cdots, m$ and $k \in \mathbb{N}$,

$$
\left\|v_{k}\right\|^{2} \leq-2 \beta_{k}\left\langle\nabla f_{i}\left(x_{k}\right), v_{k}\right\rangle \leq 2 \beta_{\max }\left\|\nabla f_{i}\left(x_{k}\right)\right\|\left\|v_{k}\right\|
$$

and thus

$$
\left\|v_{k}\right\| \leq 2 \beta_{\max }\left\|\nabla f_{i}\left(x_{k}\right)\right\| \text {. }
$$

So $\left\{v_{k}\right\}$ is bounded thanks to the boundedness of $\left\{x_{k}\right\}$ and the continuous differentiability of each $f_{i}$. Note also that $\left\{\gamma_{k}\right\} \subseteq(0,1]$. Thus, we may assume that both $\left\{x_{k}\right\}$ and $\left\{x_{k}+v \gamma_{k} v_{k}\right\}$ are contained in $\rho \mathbf{B}$ (B denotes the closed unit ball of $\mathbb{R}^{n}$ ) for some $\rho>0$, and each $\nabla f_{i}$ with $i \in\{1, \cdots, m\}$ is Lipschitz continuous on $\rho \mathbf{B}$ with Lipschitz constant $L$.

Now, we claim that, for each $i=1, \cdots, m$ and $k \in \mathbb{N}$,

$$
f_{i}\left(x_{k+1}\right) \leq f_{i}\left(x_{k}\right)-a\left\|\nabla f_{i}\left(x_{k}\right)\right\|
$$

where

$$
a=\min \left\{\frac{c \sigma}{v}, \frac{c \sigma(1-\sigma)}{L v \beta_{\max }}\right\} .
$$

Indeed, by the line search rule in (3.1), we have that for each $i=1, \cdots, m$ and $k \in \mathbb{N}$,

$$
f_{i}\left(x_{k+1}\right) \leq f_{i}\left(x_{k}\right)+\sigma \gamma_{k}\left\langle\nabla f_{i}\left(x_{k}\right), v_{k}\right\rangle \text {. }
$$

Then, (4.7) can be obtained by applying the assumption (A2) and Lemma 4.2 to (4.8).

Let

$$
b:=\frac{1}{a+\left(\rho+\left\|x^{*}\right\|\right)\left(1+2 L \beta_{\max }\right)} .
$$

In the following, to show the desired conclusion (4.5), we prove equivalently that, for each $i=1, \cdots, m$,

$$
f_{i}\left(x_{k+1}\right)-f_{i}\left(x^{*}\right) \leq(1-a b)\left(f_{i}\left(x_{k}\right)-f_{i}\left(x^{*}\right)\right) \quad \forall k \in \mathbb{N},
$$

that is, (4.5) holds with $\theta=1-a b$.

Fix $i \in\{1, \cdots, m\}$ and $k \in \mathbb{N}$. We consider two cases: (i) $\left\|\nabla f_{i}\left(x_{k}\right)\right\| \geq b\left(f_{i}\left(x_{k}\right)-f_{i}\left(x^{*}\right)\right)$, and (ii) $\left\|\nabla f_{i}\left(x_{k}\right)\right\|<b\left(f_{i}\left(x_{k}\right)-f_{i}\left(x^{*}\right)\right)$.

Assume first that (i) holds. Then, by (4.7) and the assumption (i), it is easy to obtain that

$$
\begin{aligned}
f_{i}\left(x_{k+1}\right)-f_{i}\left(x^{*}\right) & \leq f_{i}\left(x_{k}\right)-f_{i}\left(x^{*}\right)-a\left\|\nabla f_{i}\left(x_{k}\right)\right\| \\
& \leq(1-a b)\left(f_{i}\left(x_{k}\right)-f_{i}\left(x^{*}\right)\right) .
\end{aligned}
$$


Now we assume that (ii) holds. Since $f_{i}$ is convex, by the gradient inequality of $f_{i}$ and the fact that $\left\{x_{k}\right\} \subseteq \rho \mathbf{B}$, we have

$$
\begin{aligned}
f_{i}\left(x_{k+1}\right)-f_{i}\left(x^{*}\right) & \leq \nabla f_{i}\left(x_{k+1}\right)\left(x_{k+1}-x^{*}\right) \\
& \leq\left\|\nabla f_{i}\left(x_{k+1}\right)\right\|\left\|x_{k+1}-x^{*}\right\| \\
& \leq\left(\rho+\left\|x^{*}\right\|\right)\left\|\nabla f_{i}\left(x_{k+1}\right)\right\| \\
& \leq\left(\rho+\left\|x^{*}\right\|\right)\left(\left\|\nabla f_{i}\left(x_{k+1}\right)-\nabla f_{i}\left(x_{k}\right)\right\|+\left\|\nabla f_{i}\left(x_{k}\right)\right\|\right) .
\end{aligned}
$$

By using the Lipschitz continuity assumption on $\nabla f_{i}$ and the fact that $\gamma_{k} \leq 1$, one has

$$
\left\|\nabla f_{i}\left(x_{k+1}\right)-\nabla f_{i}\left(x_{k}\right)\right\| \leq L\left\|x_{k+1}-x_{k}\right\|=L \gamma_{k}\left\|v_{k}\right\| \leq L\left\|v_{k}\right\| \text {. }
$$

Applying this and (4.6) to (4.10), and taking into account the assumption (ii), we have

$$
\begin{aligned}
f_{i}\left(x_{k+1}\right)-f_{i}\left(x^{*}\right) & \leq\left(\rho+\left\|x^{*}\right\|\right)\left(1+2 L \beta_{\max }\right)\left\|\nabla f_{i}\left(x_{k}\right)\right\| \\
& \leq b\left(\rho+\left\|x^{*}\right\|\right)\left(1+2 L \beta_{\max }\right)\left(f_{i}\left(x_{k}\right)-f_{i}\left(x^{*}\right)\right) \\
& =(1-a b)\left(f_{i}\left(x_{k}\right)-f_{i}\left(x^{*}\right)\right) .
\end{aligned}
$$

Consequently, we have shown (4.9) and the proof is complete.

\section{Acknowledgments}

The research of Xiaopeng Zhao was supported in part by the National Natural Science Foundation of China (grant number 11801411).

\section{REFERENCES}

[1] H. Eschenauer, J. Koski, A. Osyczka, Multicriteria Design Optimization, Springer, Berlin, 1990.

[2] J. Fliege, OLAF-A general modeling system to evaluate and optimize the location of an air polluting facility, OR Spektrum, 23 (2001), 117-136.

[3] Y. Fu, U.M. Diwekar, An efficient sampling approach to multiobjective optimization, Ann. Oper. Res. 132 (2004), 109-134.

[4] D.T. Luc, Theory of Vector Optimization, Lecture Notes in Economics and Mathematical Systems, Springer, Berlin, 1989.

[5] D. Prabuddha, J.B. Ghosh, C.E. Wells, On the minimization of completion time variance with a bicriteria extension, Oper. Res. 40 (1992), 1148-1155.

[6] D.J. White, Epsilon-dominating solutions in mean-variance portfolio analysis, European J. Oper. Res. 105 (1998), 457-466.

[7] S. Zeng, S. Migorski, Z. Liu, J.C. Yao, Convergence of a generalized penalty method for variationalhemivariational inequalities, Commun. Nonlinear Sci. Numer. Simul. 92 (2021), 105476.

[8] L.V. Nguyen, Q.H. Ansari, X. Qin, Weak sharpness and finite convergence for solutions of nonsmooth variational inequalities in Hilbert spaces, Appl. Math. Optim. 84 (2021), 807-828.

[9] H. Zhang, W.W. Hager, A nonmonotone line search technique and its application to unconstrained optimization, SIAM J. Optim. 14 (2004), 1043-1056.

[10] L.V. Nguyen, X. Qin, Some results on strongly pseudomonotone quasi-variational inequalities, Set-Valued Var. Anal. 28 (2020), 239-257.

[11] X.P. Zhao, M.A. Köbis, On the convergence of general projection methods for solving convex feasibility problems with applications to the inverse problem of image recovery, Optimization, 67 (2018), 1409-1427.

[12] X.P. Zhao, Y.H. Yao, Modified extragradient algorithms for solving monotone variational inequalities and fixed point problems, Optimization. 69 (2020), 1987-2002.

[13] J. Fan, L. Liu, X. Qin, A subgradient extragradient algorithm with inertial effects for solving strongly pseudomonotone variational inequalities, Optimization, 69 (2020), 2199-2215. 
[14] F.U. Ogbuisi, The projection method with inertial extrapolation for solving split equilibrium problems in Hilbert spaces, Appl. Set-Valued Anal. Optim. 3 (2021), 239-255.

[15] J.Y. Bello Cruz, L.R. Lucambio Pérez, J.G. Melo, Convergence of the projected gradient method for quasiconvex multiobjective optimization, Nonlinear Anal. 74 (2011), 5268-5273.

[16] N.S. Fazzio, M.L. Schuverdt, Convergence analysis of a nonmonotone projected gradient method for multiobjective optimization problems, Optim. Lett. 13 (2019), 1365-1379.

[17] L.M. Graña Drummond, A.N. Iusem, A projected gradient method for vector optimization problems, Comput. Optim. Appl. 28 (2004), 5-29.

[18] X.P. Zhao, M.A. Köbis, Y.H. Yao, J.C. Yao, A projected subgradient method for nondifferentiable quasiconvex multiobjective optimization problems, J. Optim. Theory Appl. 190 (2021), 82-107.

[19] E.H. Fukuda, L.M. Graña Drummond, On the convergence of the projected gradient method for vector optimization, Optimization, 60 (2011), 1009-1021.

[20] J.A.d.S. Gromicho, Quasiconvex Optimization and Location Theory, Springer, US, 1998.

[21] E.A. Papa Quiroz, H.C.F. Apolinário, K.D. Villacorta, P.R. Oliveira, A linear scalarization proximal point method for quasiconvex multiobjective minimization, J. Optim. Theory Appl. 183 (2019), 1028-1052.

[22] N. Mahdavi-Amiri, F. Salehi Sadaghiani, A superlinearly convergent nonmonotone quasi-Newton method for unconstrained multiobjective optimization, Optim. Method Softw. 35 (2020), 1223-1247.

[23] S.J. Qu, Y. Ji, J.L. Jiang, Q.P. Zhang, Nonmonotone gradient methods for vector optimization with a portfolio optimization application, Eur. J. Oper. Res. 263 (2017), 356-366.

[24] R.T. Rockafellar, Convex Analysis, Priceton University Press, Priceton, 1970.

[25] R.T. Rockafellar, R.J.-B. Wets, Variational Analysis, Springer, Berlin, 1998.

[26] O.L. Mangasarian, Nonlinear Programming, McGraw-Hill, New York, 1969.

[27] S. Boyd, L. Vandenberghe, Convex Optimization, Cambridge University Press, New York, 2007.

[28] R. Burachik, L.M. Graña Drummond, A.N. Iusem, B.F. Svaiter, Full convergence of the steepest descent method with inexact line searches, Optimization, 32 (1995), 137-146.

[29] G.C. Bento, J.X. Cruz Neto, P.R. Oliveira, A. Soubeyran, The self regulation problem as an inexact steepest descent method for multicriteria optimization, Eur. J. Oper. Res. 235 (2014), 494-502.

[30] L.C. Ceng, J.C. Yao, Approximate proximal methods in vector optimization, Eur. J. Oper. Res. 183 (2007), $1-19$. 